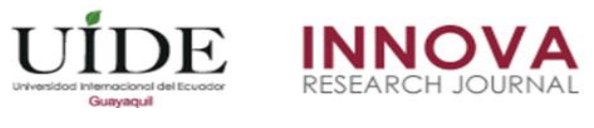

INNOVA Research Journal, ISSN 2477-9024

(Enero, 2018). Vol. 3, No.1 pp. 145-150

DOI: https://doi.org/10.33890/innova.v3.n1.2018.607

URL: http://revistas.uide.edu.ec/index.php/innova/index

Correo: innova@uide.edu.ec

\title{
Análisis y diagnóstico del sistema de control electrónico de inyección de combustible diésel HEUI CAT-3126
}

\section{Analysis and diagnosis of the electronic fuel injection control system HEUI CAT-3126}

MSc. Edwin Giovanny Puente Moromenacho

Universidad Internacional del Ecuador, Ecuador

Autor para correspondencia: epuente@uide.edu.ec

Fecha de recepción: 19 de Julio 2017 - Fecha de aceptación: 15 de Enero de 2018

Resumen: Los motores Diésel 3126 HEUI para camiones utilizan un sistema de combustible de inyectores unitarios electrónicos activados hidráulicamente. La bomba de inyección, la tubería de combustible y los inyectores que se usaban en los motores con sistema de inyección mecánica han sido remplazados con un inyector unitario electrónico hidráulico en cada cilindro. El sistema HEUI es un sistema de combustible de inyección directa a alta presión de rendimiento demostrado. Dicho sistema vigila electrónicamente los mandos del operador y la máquina para mejorar el rendimiento del motor. El sistema HEUI es exclusivo en lo que se refiere a su capacidad de controlar independientemente la presión de inyección en toda la gama de operación del motor. Esto permite un control completo de la sincronización, duración, rendimiento y eficiencia de la inyección. Dispone de una tecnología de control de inyección que modifica las características de desprendimiento de calor del proceso de combustión para disminuir significativamente los niveles de ruido y emisiones. El humo de escape se reduce considerablemente mediante un control electrónico preciso de los límites de inyección de combustible y de la sincronización de inyección del sistema HEUI. Los motores implementados con el Sistema HEUI presentan las siguientes características. Rendimiento superior. - Una nueva clase de camiones para conseguir alta producción y bajo costo por tonelada en aplicaciones de transportes en minas y canteras. Funcionamiento fiable y duradero. - Su construcción robusta y facilidad de mantenimiento garantiza una larga vida útil con bajos costos de operación.

Palabras clave: diésel, HEUI; inyección; humo; hidráulico

Abstract: The 3126 HEUI diesel engines for trucks use a fuel system of hydraulically activated electronic unit injectors. The injection pump, the fuel line and the injectors that were used in the engines with mechanical injection system have been replaced with a hydraulic electronic unit injector in each cylinder. The HEUI system is a proven high-pressure direct injection fuel system. This system electronically monitors the controls of the operator and the machine to improve the performance of the engine. The HEUI system is unique in its ability to independently control the injection pressure throughout the engine's operating range. This allows a complete control of the timing, duration, performance and efficiency of the injection. It has an injection control technology that modifies the characteristics of heat evolution of the combustion process to significantly reduce the levels of noise and emissions. The exhaust smoke is considerably reduced by precise electronic control of fuel injection limits and injection timing of the HEUI system. The motors implemented 
with the HEUI System have the following characteristics. Superior performance - A new class of trucks to achieve high production and low cost per ton in transport applications in mines and quarries. Reliable and durable operation. - Its robust construction and ease of maintenance guarantees a long service life with low operating costs.

Key words: diesel; HEUI; injection; smoke; hydraulic

\section{Introducción}

HEUI (Hidráulicamente operado Electrónicamente controlado Inyector Unitario) es el sistema de combustible que representa una de las innovaciones más significantes en la tecnología del motor diésel en décadas. HEUI supera muchas de las limitaciones de los sistemas mecánicos y de los inyectores electrónicos convencionales, y se apega a las nuevas normas para la eficacia de combustible, fiabilidad y control de emisiones.

Se lo encuentra como el equipo en motores CAT® que se adaptan fácilmente en varios tipos de vehículos y máquinas, el sistema de HEUI es muy sofisticado usa la energía hidráulica en lugar de la energía mecánica para operar los inyectores de combustible.

Trabajando en el conjuntamente con el motor, el ECM (Módulo de Control Electrónico) del sistema de HEUI proporciona mando sumamente preciso de la inyección de combustible midiéndolo y cronometrando su consumo, mientras se produce un funcionamiento del motor incomparable y con economía de combustible.

Los motores Diésel 3126 HEUI para camiones utilizan un sistema de combustible de inyectores unitarios electrónicos activados hidráulicamente. La bomba de inyección, la tubería de combustible y los inyectores que se usaban en los motores con sistema de inyección mecánica han sido remplazados con un inyector unitario electrónico hidráulico en cada cilindro. El sistema HEUI es un sistema de combustible de inyección directa a alta presión de rendimiento demostrado. Dicho sistema vigila electrónicamente los mandos del operador y la máquina para mejorar el rendimiento del motor.

El sistema HEUI es exclusivo en lo que se refiere a su capacidad de controlar independientemente la presión de inyección en toda la gama de operación del motor. Esto permite un control completo de la sincronización, duración, rendimiento y eficiencia de la inyección. Dispone de una tecnología de control de inyección que modifica las características de desprendimiento de calor del proceso de combustión para disminuir significativamente los niveles de ruido y emisiones. El humo de escape se reduce considerablemente mediante un control electrónico preciso de los límites de inyección de combustible y de la sincronización de inyección del sistema HEUI.

Los motores implementados con el Sistema HEUI presentan las siguientes características. Rendimiento superior. Una nueva clase de camiones para conseguir alta producción y bajo costo por tonelada en aplicaciones de transportes en minas y canteras. Funcionamiento fiable y duradero. - Su construcción robusta y facilidad de mantenimiento garantiza una larga vida útil con bajos costos de operación. 


\section{Materiales y Métodos}

Los HEUI que alimentan el sistema consisten en cuatro componentes básicos:

1. El inyector HEUI usa la energía hidráulica (como opuesto a la energía mecánica del árbol de levas del motor) del aceite del motor presurizándolo para la inyección. La presión del aceite entrante ( 800 a 3300 psi) proporciona el control de la inyección, mientras la cantidad de combustible inyectada es determinada por el ECM.

2. El módulo del control electrónico (ECM) es la computadora a bordo que precisamente maneja inyección de combustible y otros sistemas del motor. El solenoide del inyector HEUI da energía por una señal electrónica de 105 voltios, generados en el ECM. Las señales de entradas son proporcionadas por múltiples sensores, los microprocesadores duales del ECM usan al software interno y trabajan con un módulo de personalidad para controlar los parámetros de la actuación para producir el funcionamiento del motor máximo bajo cualquier condición.

3. La bomba de alta presión de aceite de desplazamiento constante trabaja con émbolos axiales para proporcionar el aceite inmediatamente a las salidas que necesitan de su caudal.

4. La válvula de control de la alta presión en el riel de aceite actúa electrónicamente y es operada controlada para el rendimiento de bomba de aceite y presión de la inyección.

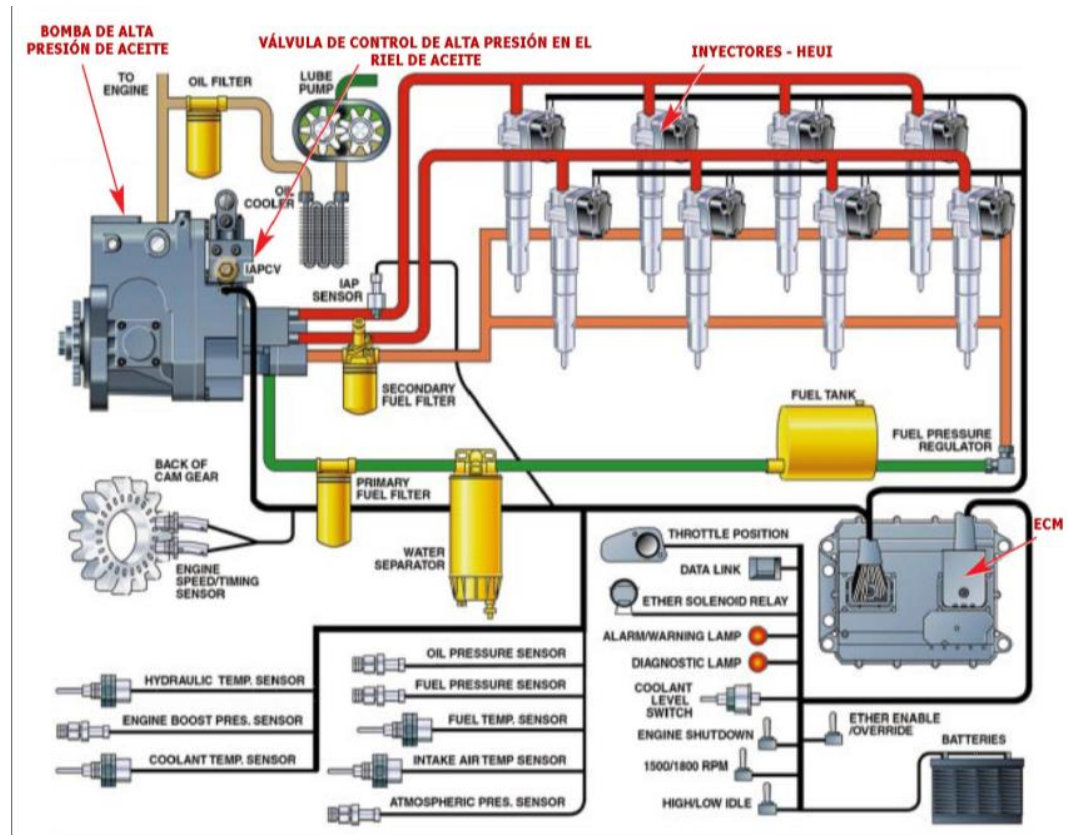

Fig 1. Partes del sistema HEUI

Fuente: (Herbert L. Nichols)

HEUI utiliza las señales de los sensores para obtener un óptimo funcionamiento del motor, sin embargo, este es un sistema que tiene cinco alimentaciones diferentes con varios 
voltajes dependiendo del sensor o del actuador si es el caso. El ECM es el elemento que proporciona este voltaje a cada uno de estos elementos.

Con esta investigación se procedió entonces a efectuar el análisis del control de los sensores, unidad de control y actuadores con la ayuda, utilizando instrumentos de medición de tecnología de punta para obtener wave forms (formas de onda) del sistema de control electrónico y su diagnóstico con las herramientas OEM establecidas.

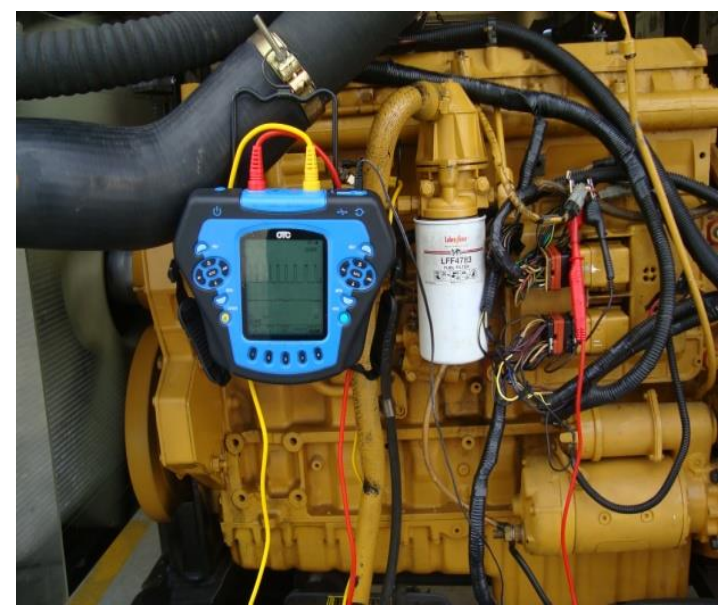

Figura 2: Toma de datos de sensores y actuadores en el motor CAT 3126 con sistema de inyección HEUI. Fuente: Pourbaix, J

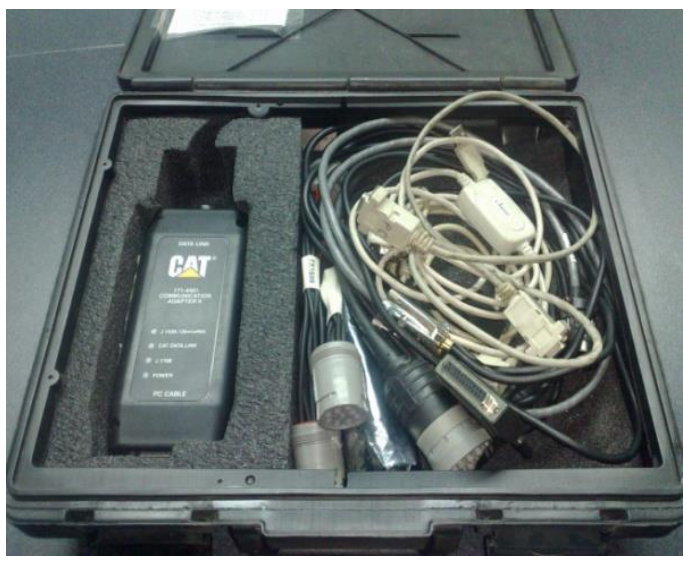

Figura 3: Kit OEM para diagnóstico de sistemas de inyección electrónica CAT. Fuente: El autor

\section{Resultados}

Se generó una tabla que contiene los valores de medición de los sensores utilizados en el diagnóstico del sistema de control electrónico CAT HEUI CAT-3126. Finalmente, se obtuvo formas de onda de onda uno de los sensores y actuadores del sistema de control electrónico CAT HEUI CAT-3126 y con sus respectivos datos PID obtenidos a lo largo de la investigación. 


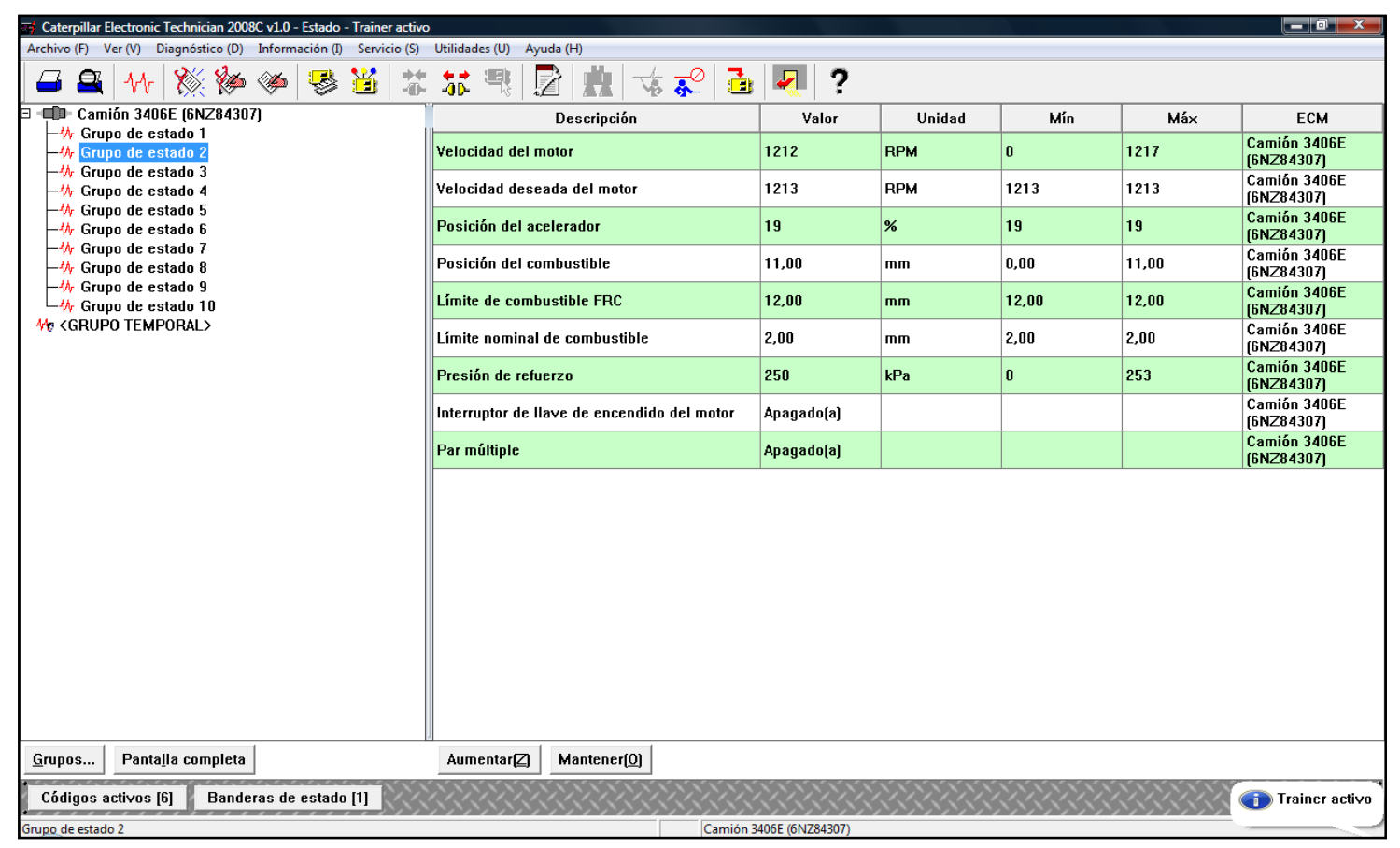

Figura 4. PID obtenidos a través del ET de CAT.

Fuente: El autor

\section{Conclusiones}

Se realizó el análisis y diagnóstico del sistema de control electrónico del sistema de inyección de combustible diésel HEUI CAT-3126, permitiendo determinar su operación, localizando averías en estos sistemas.

Se identificó el tipo de configuración y topología del sistema de inyección de combustible diésel HEUI CAT-3126.

Se analizó el diagnóstico del sistema de control electrónico del sistema de inyección de combustible diésel HEUI CAT-3126.

Se analizó el diagrama del sistema de control electrónico del sistema de inyección de combustible diésel HEUI CAT-3126.

Se utilizó instrumentos de medición de tecnología de punta para obtener wave forms (formas de onda) del sistema de control electrónico.

Se realizó una tabla que contiene los valores de medición de los sensores utilizados en el diagnóstico del sistema de control electrónico CAT HEUI CAT-3126.

\section{Bibliografía}

Angulo, J.M. (2000). Diseño Práctico De Aplicaciones - España - Mcgrow-Hill.

Dagel, J (1995). Motores Diesel Y Sistemas De Inyeccion - México - Ciencia y Técnica - 4 tomos. 
Erpelding, N.L. (1979). Reparacion Y Puesta A Punto De Motores Diesel - Barcelona Marcombo.

Gillieri, S (2004). Dispositivos Electronicos En El Automovil - S. Barcelona - Ceac - 2004

Guerber, R. (1973). Motor Diesel - Barcelona - G. Gili.

Herbert L. Nichols, Jr (2000). Manual De Reparación Y Mantenimiento De Maquinaria Pesada tomo I, II y III - Mc Graw Hill.

Miralles, J. (1982). Bombas De Inyeccion Diesel - Barcelona - CEAC.

Pla P. J.M. (1996). - Inyeccion Diesel - Barcelona - CEAC.

Pourbaix, J. (1978). Motores Diesel - Barcelona - Marcombo.

Thiessen, F. (1990). MAN 